\title{
Real-Time Power Electronics Laboratory to Strengthen Distance Learning Engineering Education on Smart Grids and Microgrids
}

\author{
Juan Roberto López Gutiérrez ${ }^{*}+\stackrel{(D)}{ }$, Pedro Ponce ${ }^{+}(\mathbb{D})$ and Arturo Molina \\ Tecnologico de Monterrey National Department of Research, Puente 222, Del. Tlalpan, \\ Mexico City 14380, Mexico; pedro.ponce@tec.mx (P.P.); armolina@tec.mx (A.M.) \\ * Correspondence: A01334289@itesm.mx \\ + These authors contributed equally to this work.
}

Citation: López Gutiérrez, J.R.; Ponce, P.; Molina, A. Real-Time Power Electronics Laboratory to Strengthen Distance Learning Engineering Education on Smart Grids and Microgrids. Future Internet 2021, 13, 237. https://doi.org/ 10.3390/fi13090237

Academic Editor: Stefano Rinaldi

Received: 27 August 2021

Accepted: 13 September 2021

Published: 17 September 2021

Publisher's Note: MDPI stays neutral with regard to jurisdictional claims in published maps and institutional affiliations.

Copyright: (c) 2021 by the authors. Licensee MDPI, Basel, Switzerland. This article is an open access article distributed under the terms and conditions of the Creative Commons Attribution (CC BY) license (https:// creativecommons.org/licenses/by/ $4.0 /)$.
Abstract: In the science and engineering fields of study, a hands-on learning experience is as crucial a part of the learning process for the student as the theoretical aspect of a given subject. With the COVID-19 pandemic in 2020, educational institutions were forced to migrate to digital platforms to ensure the continuity of the imparted lectures. The online approach can be challenging for engineering programs, especially in courses that employ practical laboratory methods as the primary teaching strategies. Laboratory courses that include specialized hardware and software cannot migrate to a virtual environment without compromising the advantages that a hands-on method provides to the engineering student. This work assesses different approaches in the virtualization process of a laboratory facility, diving these into key factors such as required communication infrastructure and available technologies; it opens a discussion on the trends and possible obstacles in the virtualization of a Real-Time (RT) laboratory intended for Microgrid education in a power electronics laboratory course, exposing the main simulation strategies that can be used in an RT environment and how these have different effects on the learning process of student, as well as addressing the main competencies an engineering student can strengthen through interaction with RT simulation technologies.

Keywords: RT laboratory; virtualization; virtual laboratory; microgrid education; distance learning

\section{Introduction}

In early 2020, the emerging COVID-19 respiratory virus stroke the world caused a complete shutdown of educational institutions in the affected countries. Educational institutions such as universities had to create new academic strategies and adapt their current educational models to meet the local government's social and health objectives, to diminish future challenges for the academic community from a health care perspective. This meant that the mission and vision of such educational institutions had to be remodeled and adapted to ensure a safe environment for their academic community; moreover, academic and educational models had to be modified as well to be congruent with the respective recommendations by the local health authorities.

The affected countries established new social and health requirement norms to more efficiently control and manage the spread of the disease; universities were forced to migrate onto digital platforms to continue with the ongoing courses offered to their student communities during the first semester of 2020 [1]. Lectures suffered very little in the virtualization process, since most of the content was already available as a digital version. However, some courses in the engineering and science faculties demand the use of laboratories and special hardware or software to reassure students' theoretical knowledge in an on-site, hands-on learning experience. These types of lectures cannot be transformed into online courses without compromising the advantages of hands-on teaching within a laboratory facility. 
For electrical engineering programs, laboratory experience is a key factor to reinforce the abstract concepts learned in a theoretical course [2]. Virtual Laboratory Setups (VLS) are a viable solution to the problems that occurred due to the suspension of on-site activities in universities. VLS provides the student with the advantages of a laboratory course during quarantine or isolating circumstances, since these can be accessed remotely from any location with a stable internet connection [3]; this characteristic is suitable for university communities with foreign students, giving them access to laboratory sessions despite their being in a remote location. This specifically applies to new educational models that offer a hybrid course option, meaning that students can choose between online, on-site, or both lecture formats for their academic courses, such as the hyflex plus Tec model developed by Tecnologico de Monterrey in Mexico [4].

Despite the need to mold the new educational strategies according to the current global pandemic, it is important to remember that universities are institutions that also work towards creating and adapting their educational framework to cover current and developing trends. For example, the growing educational demand requires the incorporation of an online education to endorse the social distance regulation, which is a mandatory factor to be considered in the new educational frameworks according to the current health requirements. In another example, universities have been working on creating and adapting their educational models to fulfill other concerning educational trends such as the integration of Sustainable Development Goals (SDG) in engineering programs, as established by the United Nations the by the year 2030 [5]. The convolution of the above examples should motivate universities to adopt new procedures when it comes to the education of future engineers, creating programs that can stay within current health regulations while promoting awareness of the paradigms that come with the integration of SDG as part of their curricula.

SDG includes affordable and clean energy generation, sustainable cities and communities, and industry innovation and infrastructure, to mention a few. The aforementioned SDG can be allocated to a single area that has been of great interest in the research community to overcome the challenges of incorporating cleaner energy generation systems into the power grid in the form of microgrids towards sustainable communities. Microgrids have many qualities that endorse the fulfillment of these SDG. For instance, microgrids can be defined as a cluster of distributed generation units, energy storage systems, dynamic loads, and a microgrid central controller to manage its operations according to desired environmental or economic objectives. Microgrids can be deployed as an independent system or with a direct connection to the power grid, providing ancillary services and helping to cover the power demand [6]. At present, microgrids are an important system in the management and control of energy generation by the inclusion of Renewable Energy Sources (RES) such as solar, wind, hydraulic, and thermal power in the electrical generation sector [7].

Microgrids have a crucial part to play as building blocks in the deployment of smartgrids and in the future of the current electrical network. Smartgrids offer a seamless combination of generation and energy management systems between renewable and nonrenewables, adding benefits such as improved power and cost relationships with enhanced energy utilities. Overall, smartgrids seek a reduction in the environmental problems caused by the energy generation sector while improving operating costs, leading to the development of a sustainable energy network.

Although microgrids are small-scale power grids in comparison to smartgrids, the goals of reliability, integration of cleaner energy generation, and the coordination and monitoring of generation and consumption assets for an optimal operation remain the same. Therefore, institutions are incorporating microgrid studies in their respective curricula to meet the 2030 SDG, recognizing the importance of having qualified electrical engineers that are capable of managing the future and present challenges of the electrical grid in the context of establishing sustainable communities [8]. 
Since power generation within independent microgrid systems is mostly carried out by distributed generation units, based on renewable energy sources, there is a need for a system that is capable of translating the obtained electrical power to a normalized energy level that can be used by the end-user. This system is commonly formed of power electronic devices and control systems. Hence, power electronics and control are considered key topics for microgrid studies in electrical engineering programs. Power electronic control laboratories are often equipped with costly and specialized equipment, meaning that users can be stranded under quarantine circumstances, representing a huge setback in the objectives in which the university invested. These laboratories can include equipment that allows for an RT simulation of a particular grid, power converters, or control models. RT simulations allow the user to assess how a simulated system would behave in a reallife scenario, leading to the establishment of a competent test-bed for an accelerated process in research into and innovation of microgrid technologies [9]. However, in the current circumstances, RT laboratories need to migrate to a virtual environment so that the advantages they offer to the learning process of the students are not negated by the current distance learning schemes.

For Wolf [10], VLS contributes to the learning process at almost an equal level to the theoretical courses of a given subject; by conducting a series of studies on certain students, it was determined that a VLS can enhance the learning of abstract concepts, while new knowledge can be acquired thanks to VLS implementation. For Soriano et al. [11], the use of specialized laboratories is mandatory for the optimal training of future engineers, acknowledging that a VLS aids in the learning process, especially when the students are located in a remote access area; thus, the authors propose a framework for a VLS, considering co-simulation, co-design, and co-modeling strategies, to enhance the process of finding solutions to complex laboratory operations, especially in facilities with RT simulation capabilities, where these simulation and validation strategies are frequently employed for microgrid and smartgrids studies.

However, since an RT simulation can be carried out in different configurations, and the tangible and intangible skills a student can strengthen are highly dependent on the laboratory arrangement, this educational aspect needs to be taken into consideration to optimize the learning process of an engineering student according to desired competences.

The work presented by Cheng and Chan [12], is a good example of how universities can still meet the 2030 goal of incorporating SDG in electrical engineering programs, since the work demonstrates an operating VLS for a power electronic course, focusing on the operation of basic DC-DC converters. Nonetheless, the conducted experiments are carried out in a non-RT scenario, and the RT simulation of the power electric circuits is not attained. In another study by Carnevali and ButtazzQ [13], a RT VLS for a robotics course at the University of Pavia is presented, allowing interaction with a RT kernel that leads to RT experiments available in the VLS through an internet connection. Finally, the work presented by Hao et al. [14], shows how a course focused on electronic and electrical experiments can be migrated to a virtual laboratory environment, offering the students a complete immersion in essential aspects of the experimental design process, from the many experimental strategies to the interpretation and analysis of the acquired data analysis.

Overall, an RT VLS is not an easy task to achieve, since the educational program has to be adapted and the students have to understand how to work in virtual environments. Limitations include the following: (i) Incorporation of specialized hardware and software, where the initial investment can be expensive; (ii) Steep learning curve when users are not familiar with simulation environments; (iii) Hardware connection and software compatibility, in cases where multiple simulation tools and environments are required; (iv) The validation of supporting communication protocols, proper communication between users and simulation platforms can be complex; (v) The computational capacity limitations of RT equipment; (vi) HIL could be useless when there are limitations in the sampling time, so an additional review has to be carried out in experimental results; (vii) The number of 
students that can run the RT simulation at the same time is limited, since RT simulation platforms can be expensive and multiuser functionality is not supported.

The aim of this work is to address the technical aspects of a virtual learning environment for an RT laboratory, aimed at undergraduate electrical engineering students. As a result of the global pandemic and the tendency of RT simulation to function as a design process, it is important to review the different aspects of translating a practical course to a virtual environment to accelerate the transition process so that the students can obtain a holistic comprehension of the technical and theoretical courses. This study incorporates the technical challenges, opportunities, and economical aspects found in the virtualization process as the possible competencies that need to be taken into account when transitioning from on-site to online teaching. This work determines key aspects of developing an RT VLS, focused mainly on a power electronics control laboratory, to offer a route map on the development of future VLS RT facilities while assessing the strengthening process of possible tangible and intangible skills for each simulation according to eligible competencies.

\section{How Have the Sustainable Development Goals Been Addressed?}

As previously mentioned, one challenge that higher educational institutions need to face is the incorporation of SDG into their current educational offers. Engineering programs need to prepare their alumni for the future challenges carried by the future power grid. The adoption of new generation systems and the evolution of the power grid to attend the SDG in affordable and clean energy generation has led to a wide range of challenges, covering a vast number of topics that need to be addressed; this suggests that universities are required to comprehend these challenges and learn how to adapt their engineering courses while applying relevant teaching methodologies and tools.

In recent years, academic institutions have introduced some courses that illustrate the environmental challenges that the current power grid carries. As an example, in the work reported by Mahmoud [15], the education regarding sustainable development in the manufacturing area suggests the incorporation of a module-based learning model, where each module is a self-contained set of information that covers specific stages and topics surrounding the sustainable aspect in a manufacturing process. Another approach was taken by Gonzalez-Pena et al. [16], where an experimental methodology was applied to motivate the student to explore and expand their own knowledge, driving the course with activities that target specific challenges of the 2030 SDG.

A complete framework based on practice-based education attacking the present issues surrounding the SDG was proposed and applied by the Swinburne University of Technology located in Melbourne Australia [17]; this novel framework introduces the students to authentic engineering problems to improve their development as professionals by working and learning simultaneously, by having a continues involvement with the end-user and experienced engineers creating professional practice habits in the students. This treats the overall learning process as a process of social involvement, not just an educational process.

Finally, a case study presented by Ponce et al. [18] introduces a novel teaching and evaluation methodology applied to a smartgrid dedicated course to help the development of professional skills among undergraduate students. The authors propose a two-sided evaluation system, where the first stage aims to solve specific problems related to the topics of the course by making use of the modeling, simulation, and manipulation of real equipment to formulate a final report on the presented problematic. Complementary to the first stage, the evaluation of the obtained reports is graded in a peer-review scheme by the student and the professor. This proposal is not only effective in the development of research-related skills, it also results in an efficient and interactive means of teaching smartgrid and microgrid topics at the undergraduate level, covering goal 7 of affordable and clean energy and goal 11 of sustainable cities and communities in the SDG scheme. Table 1 reports on the efforts of some institutions in regards to the numerous SDGs; current strategies include the development of academic programs to enlighten the teachers and 
students regarding the 2030 Agenda, the application of teaching methodologies based on a practical approach, and a theoretical analysis of the data provided by different industries to formulate optimization and control algorithms to lead certain industries in following the 2030 SDG Agenda.

Table 1. SDG addressed by different institutions.

\begin{tabular}{|c|c|c|c|c|}
\hline Institution & Country & SDG of Interest & Strategy & References \\
\hline $\begin{array}{l}\text { Swinburne University of } \\
\text { Technology }\end{array}$ & Australia & $\begin{array}{l}\text { Gender equality Inclusive } \\
\text { institutions }\end{array}$ & $\begin{array}{l}\text { Practice-based education } \\
\text { Framework }\end{array}$ & [17] \\
\hline Tecnologico de Monterrey & Mexico & Climate action & Practice-based education & [16] \\
\hline $\begin{array}{l}\text { Memorial University of } \\
\text { Newfoundland }\end{array}$ & Canada & Life below water & Program development & [19] \\
\hline Effat University & Saudi Arabia & $\begin{array}{l}\text { Affordable and clean } \\
\text { energy }\end{array}$ & $\begin{array}{l}\text { Optimization methodology in } \\
\text { electric supply chain }\end{array}$ & [20] \\
\hline $\begin{array}{l}\text { University Tenaga } \\
\text { Nasional }\end{array}$ & Malaysia & $\begin{array}{l}\text { Affordable and clean } \\
\text { energy. Sustainable cities } \\
\text { and communities }\end{array}$ & $\begin{array}{c}\text { Development of control and } \\
\text { optimization algorithms for energy } \\
\text { management systems }\end{array}$ & [21] \\
\hline University of Cádiz & Spain & $\begin{array}{l}\text { Sustainable cities and } \\
\text { communities }\end{array}$ & $\begin{array}{c}\text { Training framework for teachers } \\
\text { and students }\end{array}$ & [22] \\
\hline
\end{tabular}

It is clear that the problems induced by the incorporation of SDG in the current academic plan are not an easy to resolve; new educational frameworks need to be modeled and validated to assertively implement them as part of the curricula. Nonetheless, some of these solutions may only be viable to a certain demographic; for that reason, other solutions involving the available technological resources need to be explored. Although some technological tools can be expensive, the demographic limitations can be omitted, deriving from a homogenized educational framework.

\section{Real-Time Power Electronics Simulation Laboratory}

A power electronics laboratory equipped with RT simulation capabilities is a major upgrade from a traditional laboratory facility. Focusing on the incorporation of the SDG for 2030, Microgrid and Smartgrid studies can notably benefit from this particular simulation technology. The use of RT simulation is extensively reviewed in the literature [23,24]. The main advantages include not only a reduction in risks of real implementation, but also a reduction in the cost of extensive testing [9]. Thus, this simulation technology is ideal for educational purposes and research-oriented studies.

The RT technology attends the present issues of control techniques, distributed generation integration, grid state estimation, grid automation, protection, and high-level field-oriented applications for microgrids and Smartgrid technologies [9]. A capable laboratory can accelerate research and academic projects that foresee the resolution of the above-mentioned issues while shortening the gap between a laboratory operation and a field application.

\subsection{Microgrid}

Microgrids are considered essential for future Smartgrid implementation and in the development of sustainable communities by forcing the energy sector to innovate, finding new and better ways of incorporating Microgrids into its infrastructure as generation entities with reliable communication protocols to unify their operation towards affordable and cleaner energy production. Microgrids contribute to the 2030 SDGs by demanding generation systems capable of producing affordable and clean energy by means of RES, such as wind, solar, and hydraulic systems, opening new research and development areas for the expansion of sustainable communities, and compelling industry, innovation, and infrastructure. Figure 1 illustrates the basic concept of a microgrid system. 


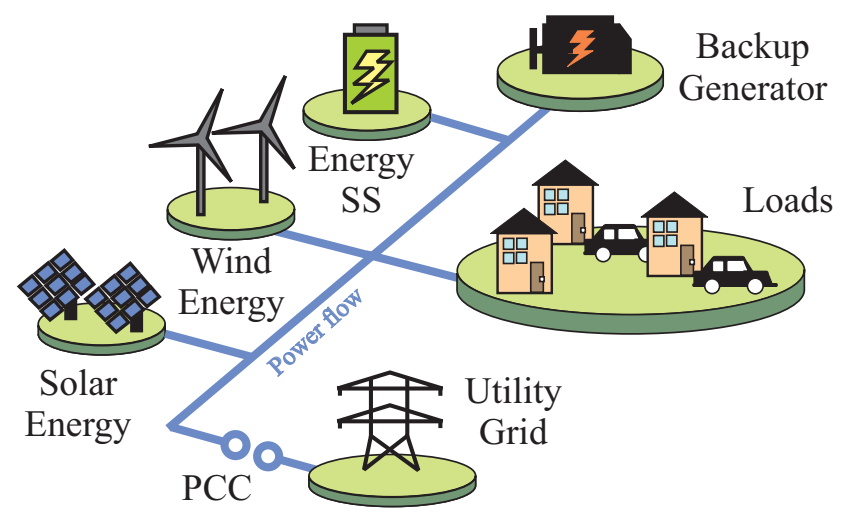

Figure 1. Microgrid system exemplification.

The addition of these RES as alternative generation units is achieved through the incorporation of power electronic devices [25]. Since the main objectives of a Microgrid are to reinforce the high penetration of the RES, straighten out the distribution grid reliability, and improve the generation performance of a sustainable power grid and islanding operation abilities of the Microgrid [26], several efforts have been made in the literature to improve its performance through the innovation and development of new control techniques for the power converters $[27,28]$. Microgrid technology development can be accelerated in an RT test environment; for example, the authors of [29] developed a laboratory prototype for voltage and frequency compensation in Microgrids with high variation in a photovoltaic generation. Another case of RT incorporation was carried out in [23], where theauthors present a fuzzy-logic-controller for a grid-connected photovoltaic system, and a Digital Signal Processor (DSP) is used for the implementation of the controller proposal in RT. As a result, the realization of a prototype is simplified and more practical. Thus, there is a need to incorporate these capable simulation technologies to fortify the academic and research departments of educational institutions.

\subsection{Real-Time}

RT simulation is used in many engineering fields. Power electronics laboratory lectures benefit from this type of simulation technology, since it can be used to simulate control and high-power level systems without risking real equipment. RT technology allows the use of new simulation techniques such as Rapid Control Prototyping, Hardware-inthe-Loop (HIL), and Hardware Under Test simulations. The benefits of this simulation technology pose a great panorama for future electrical engineers, by strengthening and helping in the development process of independent and autonomous learning, problemsolving skills, data processing, programming skills, statistical analysis, and specific industry and in-depth software familiarization.

The evolution of simulation systems is directly proportional to the availability of more capable computing technologies. Non-RT simulation methods are usually presented to engineering students; however, as RT technology becomes more available, universities are starting to include this technology as part of their engineering curricula, recognizing the advantages this technology brings to the university from a research perspective and to the students' curricula by the reinforcement of new tangent skills and competences.

Simulators work on the available computational power and are known for being dependent on the accuracy and complexity of the simulated model. RT simulations are a fixed-step simulation type, meaning that, for a given number of tasks, they must be performed at a certain time step, and its accuracy is found to be highly dependant on the length of time of each time step. To achieve RT simulation, the obtained outputs of a given number of tasks must occur before the next time step mark; if a certain task takes longer to produce the desired output than the time step, the simulation is considered erroneous; generally, this is denominated an overrun fault. Figure 2 illustrates how an RT execution is carried out as the overrun fault at a step time $C_{n}$. 


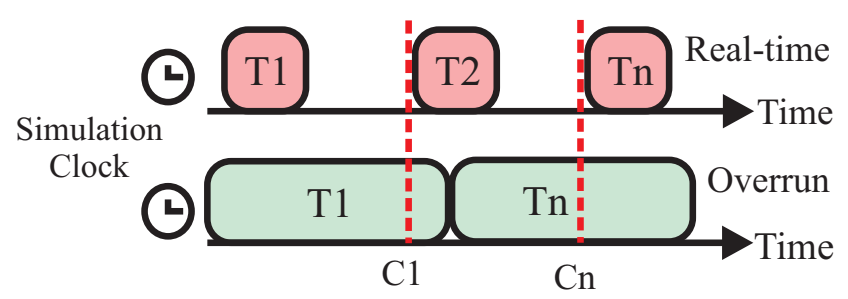

Figure 2. RT simulation example.

As mentioned previously, an RT simulation can be performed diffidently, mostly depending on the available resources; for that matter, the competencies a student can acquire can be diversified and specific to a certain RT simulation application, and further enclosed in a VLS environment. Consequently, each RT simulation type is evaluated for its ability to enhance a specific competence group, in this case, technical competencies, according to the desired internal engineering skills [30]; co-simulation and Hardware-inthe-loop are taken into consideration, since these are not hands-on restricted due to the virtual learning environment.

\subsection{Co-Simulation}

As a strategy to simulate complex and large power systems, co-simulation separates the problem into different modules and simulates each module in different RT simulators. In this manner, each simulator can independently solve a certain task, leading to more realistic behavior between modules. This strategy is an advantage for VLS, since the connection of real hardware is not necessary for its proper implementation.

One common application of the co-simulation strategy in Microgrid-oriented laboratory education is an embedded controller executing in one RT simulator, while the plant is executed in a second RT simulator. Thus, this obtains the real-life behavior and dynamics of the programmed controller over the more realistic behavior of the executed plant.

As an example, authors in [31] propose a platform between two differently geolocated RT simulators to evaluate power systems. The communication and data exchange between simulators is performed using a point-to-point User Datagram Protocol (UDP), while the user interaction is performed through an Internet connection to a public web server. Simulators are also connected to the webs server to provide the user with information about the simulator work. Another approach was taken by the authors of [32] to make use of HIL and co-simulation strategy to deliver a platform capable of delivering the detailed dynamics of a wind turbine as the behavior of the tested controllers.

\subsection{Hardware-in-the-Loop}

Laboratory courses in engineering education can include the use of specialized software and hardware. The correct manipulation and understating of hardware plays an important role in an engineer's formation. Nonetheless, studies have demonstrated that virtual and remote laboratory imputation have a greater impact on the learning outcomes when compared to traditional methods [33]. An HIL approach in a VLS can help to take advantage of both laboratory environments, traditional and virtual.

The combination of a virtual environment with the HIL simulation strategy forms a safe practice for validating hardware and software [11]. In microgrid studies, HIL is a powerful tool to test and validate embedded controllers; HIL simulation replicates the dynamic of a system according to natural circumstances in an embedded platform, leading to a more complete analysis of the simulated system ahead of the real implementation. In this manner, system and controller faults can be identified in the early stages of a project.

HIL is the next stage in the process of validating a controller's performance. The main difference between HIL and the co-simulation strategy is how the controller is implemented: in the latter, the controller is simulated in a secondary simulator, while in the former, the controller is brought down to an embedded system that reads the simulator outputs and 
computes the controller signals in an external process. The architectural difference is illustrated in Figure 3.

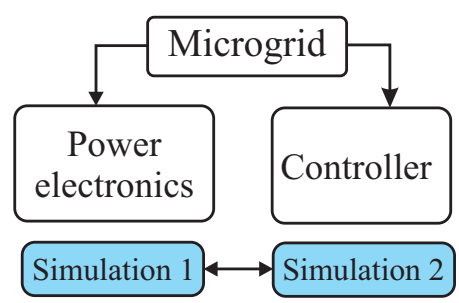

Co-simulation

(a)

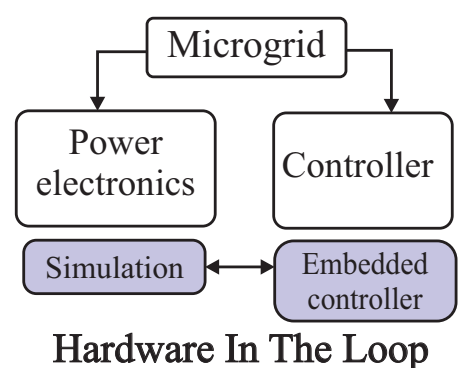

(b)

Figure 3. RT simulation strategies: (a) Co-simulation; (b) Hardware in the loop.

A combination of both simulation strategies was carried out in [32]; the authors establish an RT co-simulation platform with an HIL interface for a wind energy source. The HIL of tested controllers is achieved by using a Programmable Logic controller as an intermediate link between simulatorsl the co-simulation is carried out to model the wind turbine, while the controller is run in an HIL implementation.

\section{Competences through RT Simulation}

Competences are a central factor in the capacity of engineering graduates to seamlessly integrate with the industry. A study conducted by Najwa et al. [34], identifies the principal competencies for an engineering student, according to different countries and industries, to help in the creation of new educational strategies and shape theoretical and practical courses accordingly. Competencies can be classified as non-technical, generic, inter-disciplinary, and technical according to Male in [30]. Lim et al. [35] also defines some competencies as essential job competencies according to the importance in the working environment.

In a laboratory environment, the dominant competence group is technical skills. For engineer students, acquiring these skills is crucial for field-oriented works. Consequently, the proper identification of these technical competencies is important for their inclusion in courses that employ RT simulation technologies for a better understanding of a real-life system. Some important technical competencies, according to the different industries reviewed in [34], are: (i) application and practice of knowledge; (ii) problem solving and decision making; (iii) application of science and engineering principles; (iv) specific engineering discipline domination; (v) design and development of experiments; (vi) independent and autonomous learning; and (vii) quality and safety operation of equipment. In agreement with the studies in [24,36], where co-simulation and HIL simulation are used for an educational purpose, both RT simulation strategies concord with the need to further develop independent and autonomous learning skills, as well as problem-solving, and decision-making abilities, in future engineers. These are all defined as essential job competencies according to Lim et al. [35]. Table 2 enlists the endorsed competencies by the two different RT simulation strategies according to reviewed examples of the application in the literature [24,37]. Moreover, a definition of each skill is given below:

(i) Application and practice of knowledge: The capacity to translate theoretical knowledge into tasks through founded actions and routines. (ii) Problem solving and decision making: The capacity to analytically find solutions through gathering information to identify the optimal result among possible alternatives. (iii) Apply science and engineering principles: The capacity to apply fundamental knowledge mathematics and sciences to engineering problems. (iv) Specific engineering discipline domination: The in-depth understanding of theoretical and practical knowledge of a specific engineering field of study. (v) Design and development of experiments: The capacity to design, execute and analyze experiments. (vi) Independent and autonomous learning: The ability to self-study through the exploration of key topics to find an explanation of specific inquiries. (vii) Quality and 
safety operation of equipment: The wise use of equipment without compromising the safety of the user and others.

Table 2. Competences endorsement by RT simulation strategy.

\begin{tabular}{ccc}
\hline Competence & HIL [37] & Co-Simulation [24] \\
\hline Application and practice of knowledge & \\
Problem solving and decision making & \\
Apply science and engineering principles & \\
Specific engineering discipline domination & \\
Design and development of experiments & \\
Independent and autonomous learning & & \\
Quality and Safety operation of equipment & & \\
\hline
\end{tabular}

It is important to clarify that each simulation strategy described in Table 2 can help in the partial development of soft skills in engineering students; one would be mistaken to think that a certain skill can be developed only in the educational phase, as in the real case, where these skills are first introduced to the students, strengthened by practical and analytical courses and activities, and further developed during their work life.

As described in Table 2, HIL and co-simulation techniques are not complete methods in the development of competencies among engineering students. The competence development process, related to the specific engineering discipline domination, can be a difficult task in the HIL and co-simulation strategies, mainly because HIL and co-simulation still lack the use of real hardware, and although real system dynamics are simulated, an actual manipulation of the equipment is required to holistically dominate a specific engineering discipline, from both technical and theoretical perspectives.

On the other hand, co-simulation misses out on the design and development of experimental competence reinforcement, mainly because a higher technical knowledge is required to correctly communicate the different simulation platforms without compromising the equipment. Therefore, the participation of the student in the co-simulation strategy is limited to the use of software, without the major technical implications related to the experiments. Nonetheless, this is easily mended if the students take care of the multiple connections between simulators, crosschecking IOs ports, and software compatibility between simulator platforms to correctly design and develop experiments from a technical perspective.

\section{Final Remarks}

Currently, RT simulation technology is available through many companies. Most companies base the hardware engine on Field-Programmable Gate Array (FPGA), Intel processors, or NPX. Nonetheless, despite the differences in hardware engine architectures power electronics, HIL, control, and complex power systems simulations can still be carried out [9]. The most known simulators in the academic environment are OPAL-RT, RTDS, National Instruments, Fuji Electric, and Typhoon among others. The main difference in the implementation of a RT simulator relies on the desired software compatibility and communication interface. The former can vary from MATLAB Simulink, NI Labview, or proprietary software for a specific simulator company, i.e., RT-Lab and HYPERSIM are supported and developed for OPAL-RT hardware by OPAL-RT.

Major challenges come with the communication interface and protocols used in RT simulators; depending on the situationm this aspect could become crucial to data exchange fidelity. Some communications interfaces are only suitable for local interconnections, categorized as small-distance systems, meaning that the simulation platforms must be close to each other to ensure RT performance. On the other hand, RT simulation can be also carried out in a long-distance scenario using internet communication protocols that send pieces of data over the internet, in package form, to a specific IP address assigned to a unique simulator. In this case, simulators can be located on separate institutions, or even separate countries. Nonetheless, to ensure RT performance, the interconnected 
devices need to operate synchronously over the selected communication channel. Table 3 summarises the different communication methods used in a virtual or remote RT cosimulation environment.

Table 3. Communication interface for RT VLS.

\begin{tabular}{|c|c|c|c|c|}
\hline \multicolumn{5}{|c|}{ VLS Communication Interfaces for RT Co-Simulation } \\
\hline Interface & Advantages & Disadvantages & Small Distance & Long Distance \\
\hline Gigabit Ethernet [38] & $\begin{array}{l}\text { Inexpensive Sturdy to noise Good quality } \\
\text { in data transfer Max speed } 1 \mathrm{Gbps}\end{array}$ & Nondeterministic Not ideal for RT & & $x$ \\
\hline Optical Fiber [38] & $\begin{array}{l}\text { High volume in data transfer Secure data } \\
\text { transfer Sturdy to noise Max speed } \\
10 \mathrm{Gbps}\end{array}$ & $\begin{array}{l}\text { Fragile High cost Performance is } \\
\text { length dependent }\end{array}$ & & $x$ \\
\hline TCP/IP [39] & $\begin{array}{c}\text { Simple Cross-platform communication } \\
\text { Client-server architecture Identifiable } \\
\text { devices }\end{array}$ & $\begin{array}{l}\text { Speed } 1 \text { Mbps Not optimized for } \\
\text { local networks }\end{array}$ & & \\
\hline UDP [39] & $\begin{array}{l}\text { Simple Cross-platform Communication } \\
\text { One interface for IOs Connection-less }\end{array}$ & $\begin{array}{l}\text { Poor error detection Received data } \\
\text { can be unorganized }\end{array}$ & & \\
\hline CAN [40] & $\begin{array}{c}\text { Monitoring features Retransmission of } \\
\text { lost data } 1000 \mathrm{Kbps}\end{array}$ & $\begin{array}{l}\text { Unwanted interaction between } \\
\text { nodes High maintenance }\end{array}$ & & $x$ \\
\hline PCIe [41] & $\begin{array}{c}\text { Independent A/D channels Modular Easy } \\
\text { use Low latency }\end{array}$ & $\begin{array}{l}\text { Expensive Needs special Chassis } \\
\text { Low cross-platform compatibility }\end{array}$ & & $x$ \\
\hline
\end{tabular}

A major consideration is the corroboratation that the simulators support the same communication interface to avoid third-party linkage points, Programmable Logic Controllers (PLC) and DSP are the most popular simulators, and ensure data exchange fidelity. An important point for long-distance applications is the use of Virtual Private Networks (VPN), since the user establishes a connection to the RT simulators through the internet, meaning that a secure connection is mandatory to protect and improve the data exchange between simulators and the user.

In the case of VLS being used as a solution to migrate practical courses to online sessions due to the current global pandemic, the selection of a communication protocol capable of ensuring the long-distance manipulation of equipment is necessary. Fortunately, most of the available RT simulation technologies are built to support remote manipulation through TCP/IP and UDP communication protocols. For power electronics courses oriented for Microgrid and Smartgrid education, many simulation tools can be found in the market; these can be categorized into hardware and software tools. Table 4 shows the most popular RT simulation tools for academic and research purposes. Matlab and National Instruments software tools are commonly used by undergraduates, since these platforms require a lower initial investment, instead of the higher cost of acquiring specialized hardware and software.

Table 4. RT Hardware and Software.

\begin{tabular}{|c|c|c|c|c|c|c|}
\hline Software & Company & $\begin{array}{c}\text { Software } \\
\text { Compatiability }\end{array}$ & Long Distance & Short Distance & $\begin{array}{l}\text { Simulator Cost (USD) } \\
\text { Starting Model }\end{array}$ & License Cost (USD) \\
\hline RT-Lab & OPAL RT & Matlab LabView & & $\checkmark$ & $\$ 11,500$ & included \\
\hline Simulink RT & Matlab & Matlab & $\checkmark$ & $\checkmark$ & $\mathrm{N} / \mathrm{A}$ & $\$ 2350$ \\
\hline LabVIEW RT & $\begin{array}{c}\text { National } \\
\text { Instruments }\end{array}$ & LabVIEW & & $\checkmark$ & $\$ 3640$ & $\$ 5950$ \\
\hline RSCAD & RTDS technologies & Matlab LabVIEW & & $\checkmark$ & $\mathrm{N} / \mathrm{A}$ & $\mathrm{N} / \mathrm{A}$ \\
\hline Typhoon & Typhoon HIL & Matlab & & $\checkmark$ & $\$ 9990$ & included \\
\hline dSPACE & dSPACE & Matlab & $\checkmark$ & $\checkmark$ & $\mathrm{N} / \mathrm{A}$ & $\mathrm{N} / \mathrm{A}$ \\
\hline
\end{tabular}

Table 4 also shows the software compatibility for the different RT simulation tools; most of them are compatible with Matlab Simulink, representing an advantage for the bachelor student that is already familiarized with the software environment. Some simulators 
are also compatible with LabVIEW software, especially when incorporating GUIs and data visualization windows for the simulation.

The economical aspect of the RT simulation tools depends mostly on the objectives and preferences of the educational institution. The initial investment for entry-level equipment could be expensive; therefore, it is important to define the current and future application purposes before acquiring specialized hardware and software. This equipment could also be used to solve industrial problems. Table 4 indicates the approximate pricing of many of these simulation tools; the pricing information was obtained through official websites and available catalogs.

RT is not only defined by the simulation processing time; it is also is connected with the experimental hardware and software representation of the solution times of each subsystem and the precision of the real-time results. The minimum processing time that could be achieved depends on the manufacturer of the RT system. Table 4 is added to specify the simulation platforms that allow for long-distance manipulation in the RT system.

\subsection{Software Architecture}

The interaction between the students and laboratories is crucial for the maximum profiteering of the RT simulation resources in a laboratory course. Most VLS consist of a three-layer architecture. The first layer consists of how the student interacts and sends the information and parameters required for the laboratory interaction through a Graphical User Interface (GUI).

The second layer consists of a web server. A web server is needed to execute the client requests and to communicate with the RT simulators from a remote location. The web server is usually allocated to a different machine, since an RT simulation system cannot be used as a web server. The machine that links the simulators and the student needs to run a local web server; the most common applications to build a local webserver are python, Java, and LabVIEW Web Service. Lastly, the third layer incorporates RT simulators. This layer has the objective of initializing the simulations according to the user parameters specified in the first layer of communication. Figure 4 shows the framework of a basic communications arrangement for an RT VLS with co-simulation strategies.

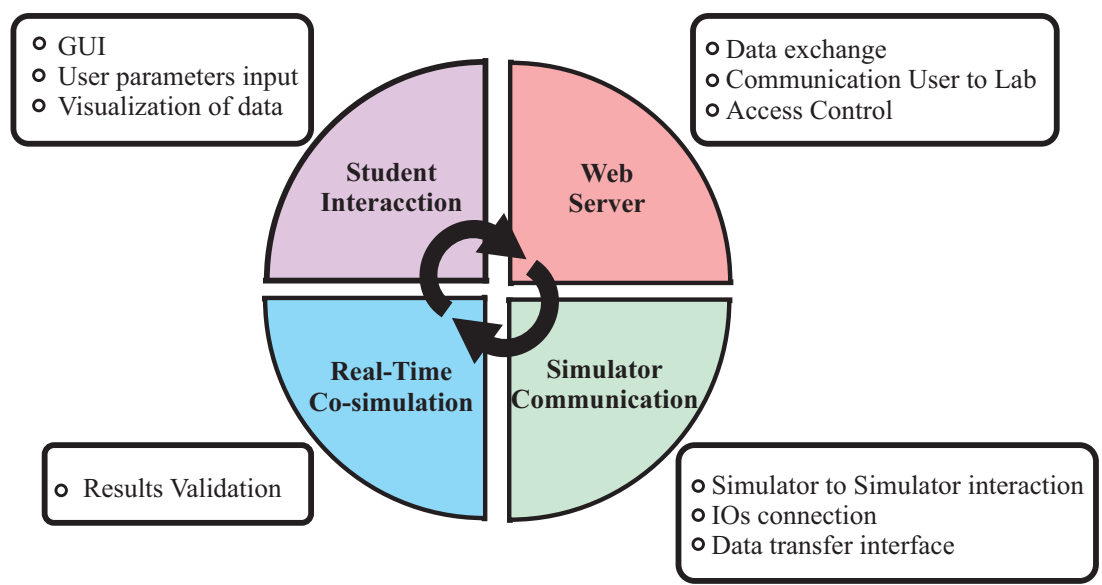

Figure 4. Framework Virtual Laboratory Setup.

A major challenge in VLS is the incorporation of new hardware into the co-simulation loop. A power electronics laboratory is usually able to facilitate a Power Hardware in the Loop (PHIL) simulation approach. PHIL is the addition of real hardware dynamics involving real power and energy transfer, and it is usually incorporated by employing a PHIL module capable of reading such power signals. The advantages of this include the analysis of the system or controller with real field-oriented hardware, making the gap between a prototype and the actual product even smaller. 
Within a VLS environment, where most students interact with pre-designed material and all modifications to a given system can be carried out through software, a PHIL approach would not be feasible; PHIL mainly operates over the manipulation physical connections, and any change in a given PHIL system requires hands-on manipulation to modify the current setup. This issue can be solved by implementing an automated laboratory capable of modifying physical connections between laboratory components. For example, an arrangement of relays operating using a PLC can be installed to modify any microgrid parameters or power converter configuration in an automated and remote manner. The work presented by Haritman et al. [42] describes the design and development of a remote laboratory equipped with PLC for the remote manipulation of equipment; the authors mention how this implementation comes with a high investment cost for the hardware. However, they also remark on the added flexibility in the practical work that can be completed by the students. It is clear that the topic of remote automated laboratories is still an open research field for further VLS development. Despite the increased cost of installation, an automated VLS would promote the students' participation by allowing for the remote manipulation of hardware. In this sense, more students can be part the laboratory courses, since all the available tools would be enclosed in virtual systems. A possible scheme for such automated configuration is illustrated in Figure 5.

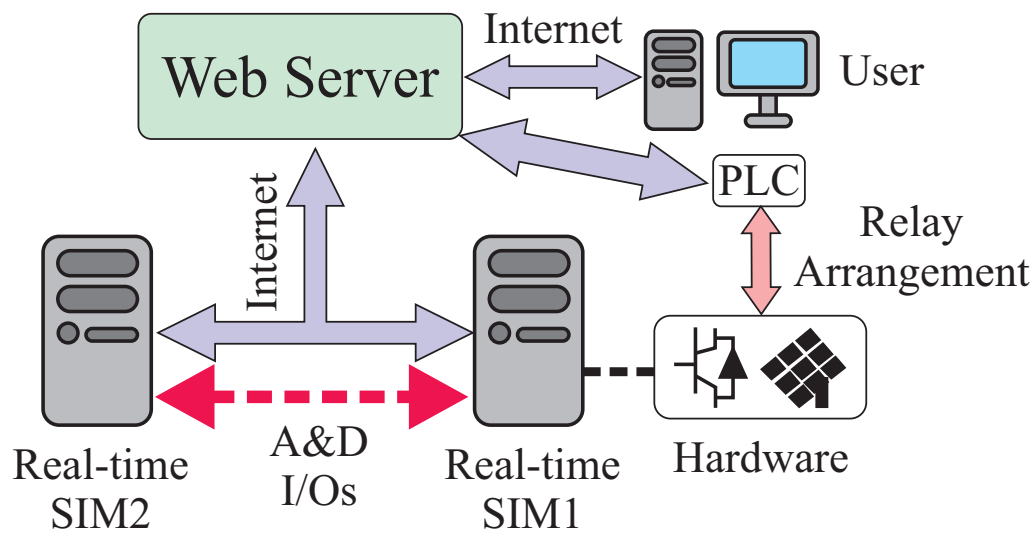

Figure 5. Architecture Virtual Laboratory Setup for PHIL applications.

\subsection{Trends in Virtual Laboratory Setups}

As the development of VLS with co-simulation and PHIL capabilities arises, the concept of Simulation as a Service (SaaS) gains strength. SaaS embraces the intention that simulators can be shared among institutions for the faster validation of results by sharing resources within different laboratories in a cloud-based environment [43]. Therefore, simulation becomes a technical service. SaaS can improve the collaboration between institutions, and strengthen the link between academic work and industries.

The linkage between institutions is also favorable to exploit professional skills among engineering students, since it requires the collaboration of multiple participants, enhancing communication and the ability to transmit knowledge in interdisciplinary and/or international scenarios. SaaS can also be used as a remunerative solution for the initial investment of RT equipment by universities, by allowing clients to make use of their facilities without human interaction and at a lower cost [44]. On the other hand, RT VLS trends include their inclusion in international educational models of engineering students; these can be used as a compelling resource to draw in international students despite the quarantine or isolating circumstances.

VLS for power electronic lectures can be further improved by adding a certain level of automation. This is a desirable characteristic due to the reduction in manual steps, such as hardware changes and the manual reordering of simulation components, resulting in the minimization of misidentification and misconnection human errors. A certain level of autonomy would allow for a more flexible remote operation, offering the ability to remotely modify the learning material and not settle for pre-defined hardware configurations. The 
concept of an automated laboratory is not new, and is highly endorsed in the medical field, since some experiments can be hazardous for hands-on manipulation [45]. Additionally, new competencies can be refined in an automated VLS, since a higher degree of knowledge is required to make hardware modifications, further enriching the education of future engineers. Nonetheless, a high level of automation would require an ever greater investment in high-fidelity communication lines, PLC, and relay systems for its implementation.

Overall, the development of RT VLS should provide institutions with the necessary tools to provide the student with a holistic learning experience, covering an area ranging from the theoretical to the practical aspects of a given subject. It would allow the student to use advantageous tools to formulate innovative solutions to the technical problem following the smartgrid development. In this sense, the student's curriculum greatly benefit's from practical experience with the Real-Time simulation technologies used in research and industry fields, which leads to an improvement in their employment and business opportunities, as the future workforce of future electrical networks.

The incorporation of RT-based learning and teaching tools is crucial due to the growing interest in novel energy network topologies such as microgrids and smartgrids. These systems come with the addition of alternative generation sources, such as wind and photovoltaic systems, to the network's generation scheme. Furthermore, the SDGs, such as goal 7 of affordable and clean energy development and goal 11 of sustainable cities and communities, have highly promoted the development of new energy network topologies. Considering that it is important to validate the current and future technologies behind these systems, RT can be contemplated as a "low-cost" and reliable solution to deal with upcoming paradigms. Moreover, RT VLS helps in the realization of these strategies in the academic sector by not limiting students and professors to theoretical courses, solving the shortcomings of the current distance-learning approach.

\section{Conclusions}

The technical aspect behind the smart grid and microgrid education cannot be fully covered by theoretical courses, and requires the modification of key features. For example, the students need to still be able to use the simulation, modeling, and hardware tools commonly found in laboratory lectures to understand specific problems in the electrical network of the future. Therefore, it is essential to comprehend how each technological tool found in a laboratory course focused on smartgrid and microgrid technologies contributes to the partial development of competencies among engineering students. This is needed to assertively create a virtual-based educational model that can fit the academic needs regarding the challenges surrounding forced remote education and SDG.

This work presents an assessment of and opens a discussion on the virtualization of a power electronics laboratory with RT capabilities as a possible strategy to overcome the impact of online courses being used in laboratory lectures as the result of quarantine or isolating circumstances, addressing the technical skills that VLS enhances in the training of future engineers. An essential point for consideration is discussed, and some limitations of RT simulation technology in a virtual environment. Communication interfaces are reviewed so that a more strategic selection of platforms can be carried out to ensure data exchange fidelity. A framework for RT VLS is introduced, based on the typical three-layer VLS architecture. The objective of each layer is described as the role it plays in a VLS. Overall, RT VLS has technical and educational trends that need to be addressed. The educational model needs to adopt new strategies to maintain the advantages of a hands-on learning education without personal interaction. While, from the technical perspective, new investments are required for a completely automated laboratory and enhance online education in laboratory courses. 
Author Contributions: Conceptualization, J.R.L.G. and P.P.; methodology, J.R.L.G. and P.P.; validation, J.R.L.G. and P.P.; investigation, J.R.L.G.; writing—original draft preparation, J.R.L.G.; writingreview and editing, J.R.L.G. and P.P.; visualization, J.R.L.G.; supervision, P.P. and A.M.; project administration, P.P. and A.M.; funding acquisition, A.M. All authors have read and agreed to the published version of the manuscript.

Funding: This research received no external funding.

Institutional Review Board Statement: Not applicable.

Informed Consent Statement: Not applicable.

Data Availability Statement: Not applicable, the study does not report any data.

Conflicts of Interest: The authors declare no conflict of interest.

\begin{tabular}{|c|c|}
\hline \\
\hline \multicolumn{2}{|c|}{ Abbreviations } \\
\hline RT & Real-Time \\
\hline VLS & Virtual Laboratory Setups \\
\hline SDG & Sustainable Development Goals \\
\hline RES & Renewable Energy Sources \\
\hline DSP & Digital Signal Processor \\
\hline HIL & Hardware in the Loop \\
\hline UDP & User Datagram Protocol \\
\hline FPGA & Field-Programmable Gate Array \\
\hline VPN & Virtual Private Network \\
\hline GUI & Graphical User Interface \\
\hline PHIL & Power Hardware in the Loop \\
\hline PLC & Programmable Logic Controllers \\
\hline SaaS & Simulation as a Service \\
\hline
\end{tabular}

\section{References}

1. Sahu, P. Closure of Universities Due to Coronavirus Disease 2019 (COVID-19): Impact on Education and Mental Health of Students and Academic Staff. Cureus 2020, 12, e7541. [CrossRef]

2. Tanyildizi, E.; Orhan, A. A virtual electric machine laboratory for effect of saturation of the asynchronous machine application. Comput. Appl. Eng. Educ. 2009, 17, 422-428. [CrossRef]

3. Kumar, D.; Radhamani, R.; Nizar, N.; Achuthan, K.; Nair, B.; Diwakar, S. Virtual and remote laboratories augment self learning and interactions: Development, deployment and assessments with direct and online feedback. PeerJ Prepr. 2018, 6, e26715v1. [CrossRef]

4. ITESM. HyFlex + Tec; ITESM: Monterrey, Mexico, 2020.

5. Ramirez-Mendoza, R.A.; Morales-Menendez, R.; Melchor-Martinez, E.M.; Iqbal, H.M.N.; Parra-Arroyo, L.; Vargas-Martínez, A.; Parra-Saldivar, R. Incorporating the sustainable development goals in engineering education. Int. J. Interact. Des. Manuf. 2020, 14, 739-745. [CrossRef]

6. Takano, H.; Goto, R.; Soe, T.Z.; Tuyen, N.D.; Asano, H. Operation Scheduling Optimization for Microgrids Considering Coordination of Their Components. Future Internet 2019, 11, 223. [CrossRef]

7. Kiptoo, M.K.; Adewuyi, O.B.; Lotfy, M.E.; Amara, T.; Konneh, K.V.; Senjyu, T. Assessing the Techno-Economic Benefits of Flexible Demand Resources Scheduling for Renewable Energy-Based Smart Microgrid Planning. Future Internet 2019, 11, 219. [CrossRef]

8. Islam, M.; Amin, M.R. Renewable-Energy Education for Mechanical Engineering Undergraduate Students. Int. J. Mech. Eng. Educ. 2012, 40, 207-219. [CrossRef]

9. Ibarra, L.; Rosales, A.; Ponce, P.; Molina, A.; Ayyanar, R. Overview of Real-Time Simulation as a Supporting Effort to Smart-Grid Attainment. Energies 2017, 10, 817. [CrossRef]

10. Wolf, T. Assessing Student Learning in a Virtual Laboratory Environment. IEEE Trans. Educ. 2010, 53, 216-222. [CrossRef]

11. Soriano, A.; Ponce, P.; Molina, A. A Novel Design of Virtual Laboratory. In Proceedings of the 2019 IEEE 20th International Conference on Research and Education in Mechatronics (REM), Wels, Austria, 23-24 May 2019; pp. 1-6. [CrossRef]

12. Cheng, K.; Chan, C. Remote Hardware Controlled Experiment Virtual Laboratory for Undergraduate Teaching in Power Electronics. Educ. Sci. 2019, 9, 222. [CrossRef]

13. Carnevali, G.; Buttazzq, G. A virtual laboratory environment for real-time experiments. IFAC Proc. Vol. 2003, 36, 31-36. [CrossRef]

14. Hao, C.; Zheng, A.; Wang, Y.; Jiang, B. Experiment Information System Based on an Online Virtual Laboratory. Future Internet 2021, 13, 27. [CrossRef] 
15. Sengupta, D.; Huang, Y.; Davidson, C.I.; Edgar, T.F.; Eden, M.R.; El-Halwagi, M.M. Using module-based learning methods to introduce sustainable manufacturing in engineering curriculum. Int. J. Sustain. High. Educ. 2017, 18, 307-328. [CrossRef]

16. Gonzalez-Pena, O.; Morán-Soto, G.; Rodríguez-Masegosa, R.; Rodríguez-Lara, B. Effects of a Thermal Inversion Experiment on STEM Students Learning and Application of Damped Harmonic Motion. Sustainability 2021, 13, 919. [CrossRef]

17. Mann, L.; Chang, R.; Chandrasekaran, S.; Coddington, A.; Daniel, S.; Cook, E.; Crossin, E.; Cosson, B.; Turner, J.; Mazzurco, A.; et al. From problem-based learning to practice-based education: A framework for shaping future engineers. Eur. J. Eng. Educ. 2020, 46, 1-21. [CrossRef]

18. Ponce, P.; Ibarra, L.; Mata, O.; Molina, A. How to develop research skills among undergraduate engineering students to tackle current ongoing topics? A Smart-Grid case study. Int. J. Electr. Eng. Educ. 2019, 58, 113-141. [CrossRef]

19. Carter, B.; Green, S.; Leeman, R.; Chaulk, N. SmartBay: Better Information-Better Decisions. In Proceedings of the OCEANS 2008, Quebec City, QC, Canada, 15-18 September 2008; pp. 1-7. [CrossRef]

20. Al-Nory, M.T. Optimal Decision Guidance for the Electricity Supply Chain Integration With Renewable Energy: Aligning Smart Cities Research With Sustainable Development Goals. IEEE Access 2019, 7, 74996-75006. [CrossRef]

21. Parvin, K.; Lipu, M.S.H.; Hannan, M.A.; Abdullah, M.A.; Jern, K.P.; Begum, R.A.; Mansur, M.; Muttaqi, K.M.; Mahlia, T.M.I.; Dong, Z.Y. Intelligent Controllers and Optimization Algorithms for Building Energy Management Towards Achieving Sustainable Development: Challenges and Prospects. IEEE Access 2021, 9, 41577-41602. [CrossRef]

22. García-González, E.; Jiménez-Fontana, R.; Azcárate, P. Education for Sustainability and the Sustainable Development Goals: Pre-Service Teachers' Perceptions and Knowledge. Sustainability 2020, 12, 7741. [CrossRef]

23. Hannan, M.A.; Ghani, Z.A.; Mohamed, A.; Uddin, M.N. Real-Time Testing of a Fuzzy-Logic-Controller-Based Grid-Connected Photovoltaic Inverter System. IEEE Trans. Ind. Appl. 2015, 51, 4775-4784. [CrossRef]

24. Strasser, T.; Stifter, M.; Andren, F.; Palensky, P. Co-Simulation Training Platform for Smart Grids. IEEE Trans. Power Syst. 2014, 29, 1989-1997. [CrossRef]

25. Rocabert, J.; Luna, A.; Blaabjerg, F.; Rodríguez, P. Control of Power Converters in AC Microgrids. IEEE Trans. Power Electron. 2012, 27, 4734-4749. [CrossRef]

26. Cortes, C.A.; Contreras, S.F.; Shahidehpour, M. Microgrid Topology Planning for Enhancing the Reliability of Active Distribution Networks. IEEE Trans. Smart Grid 2017, 9, 6369-6377. [CrossRef]

27. Huo, Y.; Gruosso, G. Hardware-in-the-Loop Framework for Validation of Ancillary Service in Microgrids: Feasibility, Problems and Improvement. IEEE Access 2019, 7, 58104-58112. [CrossRef]

28. Lucia, O.; Urriza, I.; Barragan, L.A.; Navarro, D.; Jimenez, O.; Burdio, J.M. Real-Time FPGA-Based Hardware-in-the-Loop Simulation Test Bench Applied to Multiple-Output Power Converters. IEEE Trans. Ind. Appl. 2011, 47, 853-860. [CrossRef]

29. Shi, H.; Zhuo, F.; Yi, H.; Wang, F.; Zhang, D.; Geng, Z. A Novel Real-Time Voltage and Frequency Compensation Strategy for Photovoltaic-Based Microgrid. IEEE Trans. Ind. Electron. 2015, 62, 3545-3556. [CrossRef]

30. Male, S.; Bush, M.; Chapman, E. Understanding Generic Engineering Competencies. Australas. J. Eng. Educ. 2011, 17, 147-156. [CrossRef]

31. Stevic, M.; Vogel, S.; Grigull, M.; Monti, A.; Estebsari, A.; Pons, E.; Huang, T.; Bompard, E. Virtual integration of laboratories over long distance for real-time co-simulation of power systems. In Proceedings of the IECON 2016 -42nd Annual Conference of the IEEE Industrial Electronics Society, Florence, Italy, 23-26 October 2016; pp. 6717-6721. [CrossRef]

32. Jia, F.; Cai, X.; Lou, Y.; Li, Z. Interfacing technique and hardware-in-loop simulation of real-time co-simulation platform for wind energy conversion system. IET Gener. Transm. Distrib. 2017, 11, 3030-3038. [CrossRef]

33. Brinson, J.R. Learning outcome achievement in non-traditional (virtual and remote) versus traditional (hands-on) laboratories: A review of the empirical research. Comput. Educ. 2015, 87, 218-237. [CrossRef]

34. Najwa, A.A.; Yusri, K.; Khair, N.M. Competencies of Engineering Graduates: What are the Employer's Expectations? Int. J. Eng. Technol. 2018, 7, 519. [CrossRef]

35. Lim, J.; Yoon, J.; Kim, M. Analysis of the Educational Needs Related to, and Perceptions of the Importance of, Essential Job Competencies among Science and Engineering Graduates. Educ. Sci. 2020, 10, 85. [CrossRef]

36. Osen, O.L. On the Use of Hardware-in-the-Loop for Teaching Automation Engineering. In Proceedings of the 2019 IEEE Global Engineering Education Conference (EDUCON), Dubai, United Arab Emirates, 8-11 April 2019; pp. 1308-1315.

37. Kotsampopoulos, P.C.; Kleftakis, V.A.; Hatziargyriou, N.D. Laboratory Education of Modern Power Systems Using PHIL Simulation. IEEE Trans. Power Syst. 2017, 32, 3992-4001. [CrossRef]

38. Intel Corporation. Gigabit Ethernet Technology and Solutions; Tech Report; Intel Corporation: Santa Clara, CA, USA, 2001. Available online: http:/ / www.bh-automation.com/Download/Resources/gigabit-ethernet-technology-overview.pdf (accessed on 27 August 2021).

39. Parziale, L.; Britt, T.D.; Davis, C.; Forrester, J.; Liu, W.; Matthewes, C.; Rosselot, N. TCP/IP Tutorial and Technical Overview; Tech Report; Intel Corporation: Santa Clara, CA, USA, 2006. Available online: https://www.redbooks.ibm.com/pubs/pdfs/ redbooks/gg243376.pdf (accessed on 27 August 2021).

40. Corrigan, S. Introduction to the Controller Area Network (CAN); Application Report; Texas Instruments: Dallas, TX, USA, 2016. Available online: https:/ / www.ti.com/lit/an/sloa101b/sloa101b.pdf?ts=1631630857165\&ref_url (accessed on 27 August 2021). 
41. Creta, K.; Muthrasanallur, S. The Benefits of PCI Express Architecture for Components and System; Tech Report; Intel Corporation: Santa Clara, CA, USA, 2003. Available online: https://www.intel.in/content/dam/doc/white-paper/pci-express-architecturecomponents-and-systems-paper.pdf (accessed on 27 August 2021).

42. Haritman, E.; Somantri, Y.; Wahyudin, D.; Mulyana, E. A Remote PLC Laboratory (RLab) for Distance Practical Work of Industrial Automation. IOP Conf. Ser. Mater. Sci. Eng. 2018, 306, 012034. [CrossRef]

43. Hannay, J.E.; van den Berg, T.; Gallant, S.; Gupton, K. Modeling and Simulation as a Service infrastructure capabilities for discovery, composition and execution of simulation services. J. Def. Model. Simul. Appl. Methodol. Technol. 2020. [CrossRef]

44. Bitterman, T.; Calyam, P.; Berryman, A.; Hudak, D.E.; Li, L.; Chalker, A.; Gordo, S.; Zhang, D.; Cai, D.; Lee, C.; et al. Simulation as a service (SMaaS): A cloud-based framework to support the educational use of scientific software. Int. J. Cloud Comput. 2014, 3, 177-190. [CrossRef]

45. Woodworth, A.; Pyle, A.L. Chapter 4-Sample Processing and Specimen Misidentification Issues. In Accurate Results in the Clinical Laboratory; Dasgupta, A., Sepulveda, J.L., Eds.; Elsevier: San Diego, CA, USA, 2013; pp. 35-51. [CrossRef] 